\title{
Self-emulsifying drug delivery systems as a tool to improve solubility and bioavailability of resveratrol
}

This article was published in the following Dove Press journal:

Drug Design, Development and Therapy

5 January 2016

Number of times this article has been viewed

\author{
Gehan F Balata' \\ Ebtessam A Essa ${ }^{1,2}$ \\ Hanan A Shamardl ${ }^{3,5}$ \\ Samira H Zaidan ${ }^{4}$ \\ Mohammed AS Abourehab 1,6 \\ 'Department of Pharmaceutics, \\ Faculty of Pharmacy, Umm Al-Qura \\ University, Makkah, Saudi Arabia; \\ ${ }^{2}$ Department of Pharmaceutics, \\ Faculty of Pharmacy, Tanta University, \\ Tanta, Egypt; ${ }^{3}$ Department of \\ Pharmacology, ${ }^{4}$ Department of \\ Pharmacognosy, Faculty of Pharmacy, \\ Umm Al-Qura University, Makkah, \\ Saudi Arabia; ${ }^{5}$ Department of \\ Pharmacology, Faculty of Medicine, \\ El Fayoom University, ${ }^{6}$ Department of \\ Pharmaceutics, Faculty of Pharmacy, \\ El-Minia University, Egypt
}

Abstract: Resveratrol is a nonflavonoid polyphenolic compound which has a broad range of desirable biological actions which include antioxidant, anti-inflammatory, antidiabetic, cardioprotective, and antitumor activities. However, there is concern that the bioavailability of resveratrol may limit some of its clinical utility. So, the aim of this study was to enhance the dissolution rate and oral hypoglycemic and hypolipidemic effect of resveratrol. This was achieved using self-emulsifying drug delivery system. The solubility of resveratrol was determined in various oils, surfactants, and cosurfactants. Phase diagram was plotted to identify the efficient self-emulsification regions using olive oil, Tween 80 , and propylene glycol. The prepared self-emulsifying drug delivery system formulations were tested for thermodynamic stability, emulsification efficiency, droplet size, zeta potential, and in vitro drug release. Selfemulsification time averaged 17-99 seconds without precipitation and the mean droplet sizes ranged from 285 to $823 \mathrm{~nm}$ with overall zeta potential of -2.24 to $-15.4 \mathrm{mv}$. All formulations improved drug dissolution in relation to unprocessed drug with a trend of decreased dissolution parameters with increasing oil content. The optimized formula, F19, with dissolution efficiency of $94 \%$ compared to only $42 \%$ of pure drug was used to study the in vivo hypoglycemic and hypolipidemic effects of resveratrol in diabetic-induced albino rats and comparing these effects with that of pure resveratrol in different doses. Treatment with the optimized formula, F19, at $10 \mathrm{mg} / \mathrm{kg}$ had significant hypoglycemic and hypolipidemic effects in diabetic-induced albino rats which were nearly similar to the high dose $(20 \mathrm{mg} / \mathrm{kg})$ of unprocessed resveratrol. From the study, it was concluded that formulation F19 has good emulsification property with uniform globule size, satisfactory in vitro drug release profile, and significant in vivo hypoglycemic effects which identify future opportunities for resveratrol delivery.

Keywords: resveratrol, self-emulsifying, olive oil, Tween 80, propylene glycol

\section{Introduction}

The use of plants as medicines has a long history in the treatment of various diseases. To date, approximately 35,000-70,000 plant species have been screened for their medicinal use. ${ }^{1}$ Resveratrol (3,5,4'-trihydroxystilbene), a naturally occurring polyphenol, has attracted considerable interest for its beneficial potentials for human health, which include antioxidant, anti-inflammatory, cardioprotective, anti-hyperlipidemic, and antitumor activities. ${ }^{2-5}$ In addition, resveratrol has emerged as a leading candidate as an antidiabetic agent. Trees such as eucalyptus and spruce have been found to contain resveratrol; however, its presence in edible plants is rare. Major dietary sources include grapes, wine, peanuts, and soy. While peanuts and grapes contain low levels of resveratrol, red wine supplies relatively high concentrations of resveratrol. ${ }^{6}$ The last decades have witnessed a rising interest in resveratrol by health professionals. This is due to the fact that prolonged administration of some agents to treat hyperglycemia may induce unwanted side effects. In this context, a compound reducing blood glucose
Correspondence: Gehan F Balata Faculty of Pharmacy, Umm Al-Qura University, El-Abdia, Makkah, Saudi Arabia

Tel +966541841800

Fax+966255070424225

Email jfsyed@uqu.edu.sa submit your manuscript | www.dovepress.com

Dovepress

http://dx.doi.org// 0.2147/DDDT.S95905
Drug Design, Development and Therapy 2016:10 117-128 117

(c) (i) (5) 2016 Balata et al. This work is published and licensed by Dove Medical Press Limited. The full terms of this license are available at https://www.dovepress.com/terms.php BY and incorporate the Creative Commons Attribution - Non Commercial (unported, v3.0) License (http://creativecommons.org/licenses/by-nc/3.0/). By accessing the work you Press limited, provided the work is properly attributed. For permissio for commercial use of this work, please see paragraphs 4.2 and 5 of our Terms (https://www.dovepress.com/terms.php). 
level without any unwanted side effects, even if administered for a long period of time, would be very useful. ${ }^{7}$

It was reported that resveratrol reduces hyperglycemia by increasing insulin sensitivity in humans. ${ }^{8}$ The preventive and anti-hyperglycemic effect of resveratrol is thought to be complex and involves different effects that include stimulatory action on intracellular glucose transport and induced effects that may contribute to the protection of $\beta$-cells in diabetes. Interestingly, in experiments on isolated cells, resveratrol was able to stimulate glucose uptake in the absence of insulin. ${ }^{8}$ It was reported that peak plasma level of resveratrol after ingestion of a dose of $5 \mathrm{~g}$ was $539 \pm 384 \mathrm{ng} / \mathrm{mL}(2.4 \mathrm{mmol} / \mathrm{L}$, mean \pm standard deviation $[\mathrm{SD}] ; \mathrm{n}=10$ ), which occurred 1.5 hours postdose. This low plasma level may be due to avid metabolism of resveratrol in humans. ${ }^{9}$

The hydrophobicity of resveratrol is due to the polyphenolic structure, which makes it practically insoluble in water ( $3 \mathrm{mg} / 100 \mathrm{~mL})$ according to the European Pharmacopeia definition, with a $\log \mathrm{P}$ of $3.1 .{ }^{10}$ Despite this poor water solubility, resveratrol exhibits high membrane permeability and can be considered as a Class II compound in the Biopharmaceutical Classification System. ${ }^{11}$ Being highly permeable, enhancement of its aqueous solubility and/or dissolution rate would be a good approach to improve bioavailability by increasing the amount of drug available for absorption. In recent years, several studies have focused on novel formulation approaches to improve resveratrol bioavailability including complexation with $\beta$-cyclodextrins, ${ }^{12}$ resveratrolloaded Ca-pectinate beads, ${ }^{13} \beta$-cyclodextrin nanosponge ${ }^{3}$ and solid lipid nanoparticles, ${ }^{13}$ and liposomes. ${ }^{14}$

The technique of self-emulsifying drug delivery system (SEDDS) has been recently developed to enhance the solubility and bioavailability of poorly aqueous soluble drugs. SEDDS represents a possible alternative to traditional oral formulations of lipophilic compounds. It can be described as isotropic solutions of oil, surfactant, cosurfactant, and drug which form oil-water (o/w) emulsions when exposed to the fluids and motility of the gastrointestinal tract. This spontaneous formation of an emulsion presents the drug in a solubilized form, and the small size of the formed droplet provides a large interfacial surface area for drug absorption. ${ }^{15,16}$ Apart from solubilization, the oral bioavailability augmentation is also achieved by promotion of intestinal lymphatic transport and hence reduction in first pass metabolism, enhancement of intestinal permeability, and reduced metabolism and P-glycoprotein-mediated efflux by the incorporated surfactants such as polysorbate $80 .{ }^{16}$
The aim of this work is to prepare SEDDS of resveratrol and investigate the potential of this system to improve the dissolution rate. Optimal formulation regarding enhanced dissolution rate will be used to investigate its hypoglycemic and hypolipidemic effects in vivo.

\section{Methods \\ Materials}

Resveratrol was purchased from Xian Lukee Bio-Tech Co., Ltd., (Xi'an, Shaanxi Sheng, People's Republic of China). Oleic acid, Tween 80, Tween 20, and propylene glycol were purchased from Merck Specialities Pvt. Ltd., Mumbai, India. PEG 400, n-butanol, soybean oil, olive oil, and almond oil were purchased from SD Fine chemicals, Mumbai, India. Streptozotocin (STZ) was purchased from Sigma-Aldrich Chemical Co., Steinheim, Germany. Hydroxypropyl methyl cellulose was procured from Loba Chemie, Mumbai, India. All other chemicals were of analytical reagent grade.

\section{Saturation solubility study}

The solubility of resveratrol in various oils, surfactants, and cosurfactants was determined in $5 \mathrm{~mL}$ of selected vehicles in capped vials containing excess of drug $(50 \mathrm{mg})$. The mixtures were sonicated (Ultrasonic model SS101H, Sonix IV, Huntington Beach, CA, USA) at $40^{\circ} \mathrm{C}-50^{\circ} \mathrm{C}$ to facilitate the solubilization. Then, the mixtures were transferred to a thermostatic shaker water bath (LSB-030S, Daihan Labtech Co. Ltd., New Delhi, India) maintained at $25^{\circ} \mathrm{C}$ for 48 hours. After reaching equilibrium, vials were centrifuged (NF 815, Nuve, Ankara, Turkey) at 4,000 rpm for 10 minutes and followed by filtration through membrane filter (\# $0.45 \mu \mathrm{m})$. The clear filtrate was diluted with methanol and was measured spectrophotometrically (Shimadzu UV-160A Spectrophotometer, Shimadzu, Kyoto, Japan) at 304 nm. $^{3}$

\section{Construction of ternary phase diagram and self-emulsification assessment}

Based on the results of the saturation solubility studies, olive oil, Tween 80 (surfactant), and propylene glycol (cosurfactant) were selected to construct the ternary phase diagram. A series of formulations were prepared using varying concentrations of oil $(10 \%, 15 \%, 20 \%$, and $30 \%)$. Surfactant and cosurfactant $\left(\mathrm{S}_{\text {mix }}\right)$ at each oil percentage were mixed at different weight ratios $(1: 1,2: 1,3: 1,4: 1,1: 2$, $1: 3$, and $1: 4$, respectively). These $S_{\text {mix }}$ ratios were chosen in increasing concentration of surfactant with respect to cosurfactant and vice versa (Table 1). Doing so, 28 formulations 
Table I Composition and visual assessment of different selfemulsifying drug delivery system formulations

\begin{tabular}{lllll}
\hline Formula & \multicolumn{3}{l}{ Components $(\mathbf{m g})$} & Visual \\
\cline { 2 - 4 } & Oil & Tween $\mathbf{8 0}$ & $\begin{array}{l}\text { Propylene } \\
\text { glycol }\end{array}$ & \\
\hline observation \\
\hline FI & 100 & 450 & 450 & Good \\
F2 & 100 & 600 & 300 & Good \\
F3 & 100 & 675 & 225 & Good \\
F4 & 100 & 720 & 180 & Good \\
F5 & 100 & 300 & 600 & Good \\
F6 & 100 & 225 & 675 & Good \\
F7 & 100 & 180 & 720 & Good \\
F8 & 150 & 425 & 425 & Good \\
F9 & 150 & 566.7 & 283.3 & Good \\
FI0 & 150 & 637.5 & 215.5 & Good \\
FII & 150 & 680 & 170 & Good \\
FI2 & 150 & 283.3 & 566.7 & Good \\
FI3 & 150 & 215.5 & 637.5 & Good \\
FI4 & 150 & 170 & 680 & Good \\
FI5 & 200 & 400 & 400 & Bad \\
FI6 & 200 & 533.3 & 266.7 & Bad \\
FI7 & 200 & 600 & 200 & Good \\
FI8 & 200 & 640 & 160 & Good \\
FI9 & 200 & 266.7 & 533.3 & Good \\
F20 & 200 & 200 & 600 & Good \\
F2I & 200 & 160 & 640 & Good \\
F22 & 300 & 350 & 350 & Bad \\
F23 & 300 & 466.7 & 233.3 & Bad \\
F24 & 300 & 525 & 175 & Bad \\
F25 & 300 & 560 & 140 & Good \\
F26 & 300 & 233.3 & 466.7 & Good \\
F27 & 300 & 175 & 525 & Good \\
F28 & 300 & 140 & 560 & Good \\
\hline & & & &
\end{tabular}

were obtained and prepared by mixing the specified weight of each component in small glass vials and then vortex mixed until a clear solution was obtained. The mixtures were stored at room temperature until used.

Evaluation of the self-emulsifying properties of SEDDS formulations was performed by visual assessment. Exactly measured $0.2 \mathrm{~mL}$ of each formulation was introduced into $100 \mathrm{~mL}$ of water in a glass beaker at $37^{\circ} \mathrm{C}$ and the contents were mixed gently with a magnetic stir bar. Different formulations were categorized based on speed of emulsification, clarity, and apparent stability of the resultant emulsion. The formulations were then scored as "good" when the droplets spread easily in water without any further coalescence within 1 minute of addition and produced a clear emulsion with a bluish tinge and were judged "bad" when there was poor or no emulsion formation with immediate coalescence of oil droplets. ${ }^{17}$ Phase diagram was constructed by identifying the good self-emulsifying region using custom design software.

\section{Formulation of resveratrol SEDDS}

Resveratrol, at a concentration of $20 \mathrm{mg} / 1 \mathrm{~g}$ of each formulation, was dissolved in propylene glycol at $45^{\circ} \mathrm{C}$ in an isothermal water bath. After cooling, a calculated amount of oil and Tween 80 was added and vortexed until a clear solution was obtained. The formulation was equilibrated at ambient temperature for at least 48 hours to examine for any sign of turbidity or phase separation. ${ }^{18}$

\section{Characterization of SEDDS}

\section{Thermodynamic stability studies}

The thermodynamic stability was performed using centrifugation and accelerated aging (freeze thawing) methods. Formulations showed "good" dispersion properties were centrifuged (REMI, Mumbai, India) at the 5,000 rpm for 30 minutes and observed for any sign of phase separation, such as creaming or cracking. For freeze thawing test, only formulations that survived after the centrifugation test were selected. Test was conducted by subjecting each formulation to three to four freeze-thaw cycles, which included freezing at $-4^{\circ} \mathrm{C}$ for 24 hours followed by thawing at $40^{\circ} \mathrm{C}$ for 24 hours. $^{17}$

\section{Emulsification time and precipitation assessment}

The emulsification time of the selected stable SEDDS formulations was determined using USP dissolution tester (SP6-400, GB CALEVA Ltd., Reading, Berkshire, UK). The SEDDS formulation equivalent to $20 \mathrm{mg}$ of resveratrol was added drop wise to $500 \mathrm{~mL}$ of distilled water maintained at $37^{\circ} \mathrm{C} \pm 0.5^{\circ} \mathrm{C}$. Gentle agitation was provided by a paddle rotating at $50 \mathrm{rpm}$. The emulsification time was recorded manually. ${ }^{19}$

Precipitation was evaluated by visual inspection of the resultant emulsion after 24 hours. The formulations were then categorized as clear (transparent or transparent with bluish tinge), nonclear (turbid), stable (no precipitation at the end of 24 hours), or unstable (showing precipitation within 24 hours). ${ }^{20}$

\section{Determination of droplet size and zeta potential}

The droplet size of the emulsion is a crucial factor in the performance of self-emulsification system because it determines the rate and extent of drug release and absorption. ${ }^{21}$ The mean size and zeta potential of emulsion globules were determined by Zetasizer (Malvern Instruments, Malvern, UK) based on dynamic laser scattering. The dispersed formulations were measured after dilution $(1: 1000 \mathrm{v} / \mathrm{v})$ with distilled water and mixing for 1 minute with Cyclo mixer. 


\section{In vitro release studies}

The in vitro release of resveratrol from the stable SEDDS formulations and unprocessed drug, as control, was performed using USP Dissolution Tester Apparatus II in $900 \mathrm{~mL}$ distilled water maintained at $37^{\circ} \mathrm{C} \pm 0.5^{\circ} \mathrm{C}$. From each SEDDS formulation, an amount equivalent to $20 \mathrm{mg}$ of the drug was filled in hard gelatin capsule and placed in the dissolution vessel and the paddle was adjusted to rotate at $100 \mathrm{rpm}$. Samples of $5 \mathrm{~mL}$ were withdrawn at predetermined interval, filtered through membrane filter of $0.45 \mu \mathrm{m}$ pore size, and spectrophotometrically measured for resveratrol. The removed samples were replenished with equal volumes of the medium to keep the dissolution volume constant. Cumulative amount of resveratrol dissolved after 1 hour was used to compare between different investigated formulations.

\section{In vivo study}

\section{Experimental animals}

All animal care and procedures were conducted in accordance with the Guiding Principles in the Use of Animals in Toxicology, as adopted in 1989, revised in 1999, and amended in 2008 by the Society of Toxicology. ${ }^{22}$ Moreover, approval to carry out an in vivo study was obtained from the Institutional Animal Ethical Committee, Faculty of Pharmacy, Umm Al-Qura University, and their guidelines were followed for the study. The study used male albino rats of Wistar strain weighing 180-220 g and 6-weeks-old, maintained in clean, polypropylene cages, and fed ad libitum standard chow and water. The rats were acclimatized for 1 week and randomly divided into six groups, each group comprising of ten rats.

\section{Induction of experimental diabetes}

Diabetes was induced by a single intraperitoneal injection of STZ (50 mg/kg body weight) dissolved in $0.1 \mathrm{M}$ of cold citrate buffer ( $\mathrm{pH}=4.5$ ), after fasting for 12 hours. Rats were supported for the next 24 hours with $5 \%$ glucose solution after STZ administration to avoid STZ-induced fatal hypoglycemia as a result of massive pancreatic insulin release. After 72 hours, rats with fasting blood glucose concentration over than $250 \mathrm{mg} / \mathrm{dL}$ were selected for the experimental model of diabetes.

\section{Experimental design}

In all, 60 rats were divided randomly into six groups $(n=10)$ and treated immediately after diabetes confirmed as follows.

Group 1: Normal control rats treated with 1\% hydroxypropyl methylcellulose solution; Group 2: Diabetic rats treated by a vehicle as positive control (DM); Groups 3 and 4: Diabetic rats treated with $10 \mathrm{mg} / \mathrm{kg} /$ day of unprocessed and optimal resveratrol formula F19 (CRV10 and FRV10 groups, respectively); and Groups 5 and 6: Diabetic rats treated with $20 \mathrm{mg} / \mathrm{kg} /$ day of unprocessed and optimal resveratrol formula F19 (CRV20 and FRV20 groups, respectively). Treatment was done by oral gavage and lasted for 4 weeks. Amounts of 10 and $20 \mathrm{mg}$ resveratrol $/ \mathrm{kg}$ were selected on the basis of previous studies. $^{23,24}$ Body weight and serum glucose level were monitored weekly. At the end of the study, the rats were fasted overnight with free access to water, anesthetized, and sacrificed. Blood samples were directly collected from heart. Serum glucose, triglycerides, and high density lipoproteincholesterol (HDL-C) levels were determined enzymatically. Serum glucose was determined by glucose oxidase method (Crumlin, Country Antrim, UK). Cholesterol, HDL, and triglycerides were determined by enzymatic method using Wako test kit (Code No 274-46401; Wako Pure Chemical Industries, Ltd., Osaka, Japan). Low density lipoproteincholesterol (LDL-C) level was calculated by the formula of Friedewald et al. ${ }^{25}$ The obtained data were used to verify the hypoglycemic and hypolipidemic properties of resveratrol as well as to compare the extent of effect of both pure drug and its new formula at different therapeutic doses.

\section{Statistical analysis}

All results were expressed as mean \pm SD. Data obtained were statistically analyzed using one-way analysis of variance using SPSS 16.0; the difference at $P<0.05$ was considered to be statistically significant. In addition, release data for the formulations F19-F21 containing different $\mathrm{S}_{\text {mix }}$ ratios, droplet size, and zeta potential were subjected to bi-variant correlation.

\section{Results}

\section{Saturation solubility}

Results from solubility studies are shown in Table 2. As per the observed solubility value, olive oil showed the highest solubilization capacity for resveratrol. Regarding surfactants, the drug was more soluble in Tween 80. Among the tested cosurfactants, propylene glycol was the most appropriate.

\section{Phase diagram and self-emulsification assessment}

Figure 1 represents the phase diagram of olive oil-Tween 80 propylene glycol systems in different ratios where the dotted area represents the self-emulsification region. Translucent yellow homogeneous formulations without any visible 
Table 2 Solubility profile of resveratrol at different oils, surfactants, and cosurfactants

\begin{tabular}{ll}
\hline Vehicle & Solubility $(\mathrm{mg} / \mathrm{mL})$ \\
\hline Oleic acid & $0.326 \pm 0.075$ \\
Olive oil & $5.228 \pm 0.23$ \\
Soybean oil & $4.004 \pm 0.63$ \\
Almond oil & $4.066 \pm 0.54$ \\
Tween 80 & $9.809 \pm 0.67$ \\
Tween 20 & $8.084 \pm 0.58$ \\
Propylene glycol & $9.228 \pm 0.28$ \\
PEG 400 & $8.111 \pm 0.83$ \\
$n$-Butanol & $8.084 \pm 0.37$ \\
\hline
\end{tabular}

Note: Data are presented as mean \pm standard deviation.

particulates were obtained. SEDDS form fine o/w emulsions with only gentle agitation upon introduction into aqueous media. The incorporation of higher amounts of cosurfactant (1:3 and $1: 4 \mathrm{w} / \mathrm{w}$ surfactant:cosurfactant, respectively) within the self-emulsifying region increased the spontaneity of the self-emulsification process. The efficiency of emulsification increased as the $\mathrm{S}_{\text {mix }}$ ratio was increased and formulations containing more than $20 \%$ oil showed poor self-emulsifying properties with the formation of less clear emulsion.

\section{Characterization}

\section{Thermodynamic stability studies}

Thermodynamic stability of the selected formulations was evaluated by centrifugation and freeze thaw cycle stress tests.
All formulations passed the thermodynamic stability tests, except formulations F17, F25, and F28 which showed phase separation after centrifugation.

\section{Emulsification time and precipitation assessment}

The results of self-emulsification and precipitation studies are given in Table 3. The evaluation of emulsification time showed that with the increase in the proportion of olive oil in the formulation, the emulsification time increases. For example, at the same $\mathrm{S}_{\text {mix }}$ concentration (1:3), increasing oil concentration from $10 \%$ (F6) to $30 \%$ (F27) resulted in approximately a fivefold increase in emulsification time. To estimate the effect of $\mathrm{S}_{\text {mix }}$ concentration on emulsification time, for formula F4 through F7 prepared with $10 \%$ oil, as the concentration of surfactant decreased, the emulsification time increased. F4 (surfactant:cosurfactant ratio of $4: 1 \mathrm{w} / \mathrm{w}$ ) showed an emulsification time of 17 seconds compared to 29 seconds by F7 (1:4 w/w). Regarding drug precipitation, all formulations, except F1, F2, and F18, did not show any precipitation of drug or turbidity upon dilution and storage at room temperature for 24 hours.

\section{Determination of droplet size and zeta potential}

The results of droplet size determination of various formulations are given in Table 3. It is clear that an increase in the percentage of the oil phase led to an increase in droplet size. Keeping $\mathrm{S}_{\text {mix }}$ constant at 1:3, the droplet size in $\mathrm{F} 6$ containing

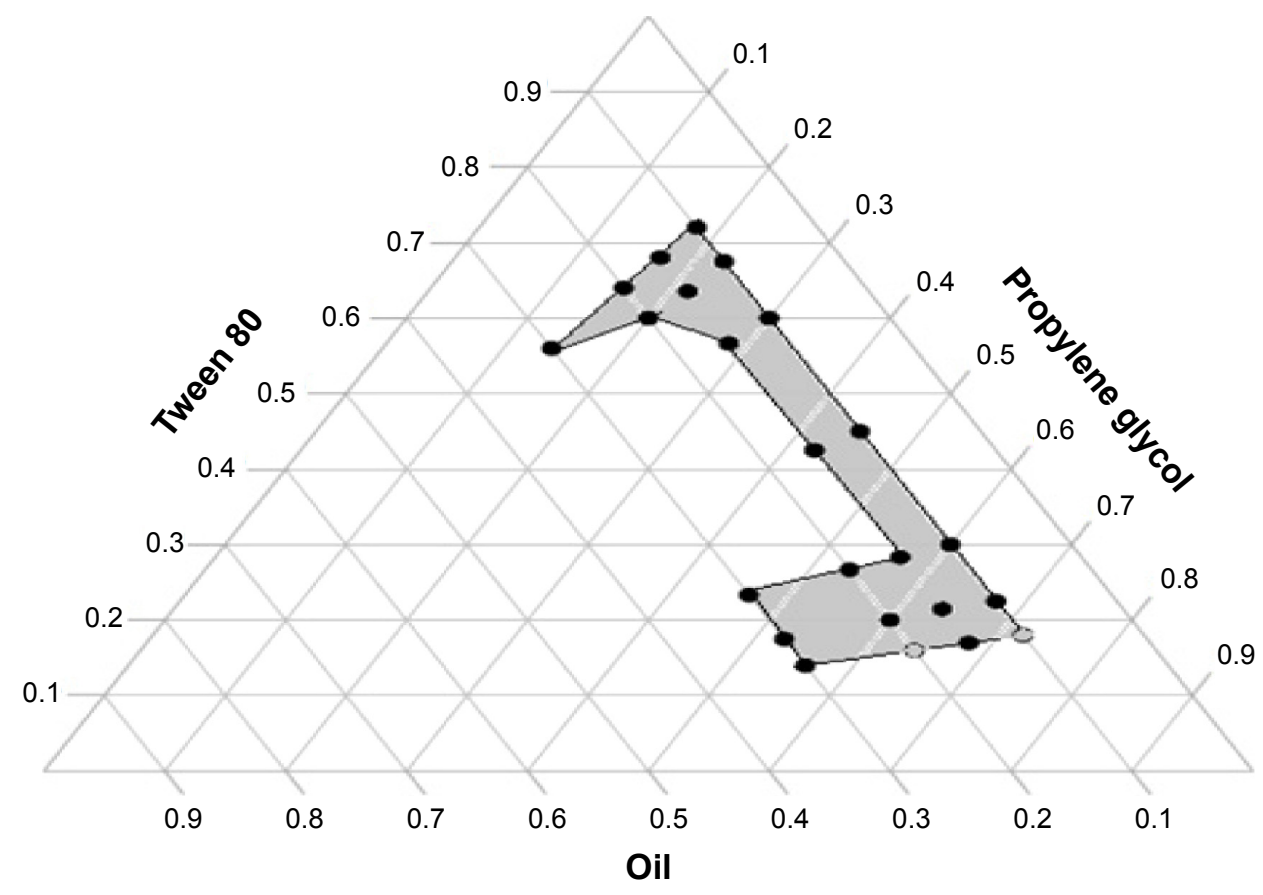

Figure I Ternary phase diagram of mixture consisting of olive oil, propylene glycol, and Tween 80. 
Table 3 Emulsification time, mean droplet size, zeta potential, percentage drug released after 5 minutes (Q5) and 60 minutes (Q60), and percentage dissolution efficiency (\%DE) of various formulations of self-emulsifying drug delivery system

\begin{tabular}{|c|c|c|c|c|c|c|}
\hline Formula & $\begin{array}{l}\text { Emulsification } \\
\text { time (s) }\end{array}$ & $\begin{array}{l}\text { Mean droplet } \\
\text { size }(\mathrm{nm})\end{array}$ & $\begin{array}{l}\text { Zeta potential } \\
(\mathrm{mV})\end{array}$ & Q5 & Q60 & \%DE \\
\hline F3 & $20 \pm 1.3$ & $440 \pm 9.9$ & $-9.73 \pm 0.16$ & $76 \pm 1.6$ & $88.0 \pm 2.1$ & 86.5 \\
\hline F4 & $17 \pm 0.5$ & $285 \pm 2.6$ & $-13.7 \pm 0.2$ & $90 \pm 2.4$ & $95.2 \pm 1.1$ & 89.6 \\
\hline F5 & $20 \pm 1.1$ & $404 \pm 5.6$ & $-8.76 \pm 0.12$ & $75 \pm 2.1$ & $90.7 \pm 2.0$ & 76.7 \\
\hline F6 & $20 \pm 0.9$ & $420 \pm 4.9$ & $-5.7 \pm 0.1$ & $80 \pm 1.7$ & $88.7 \pm 1.8$ & 80.7 \\
\hline F7 & $29 \pm 1.3$ & $459 \pm 5.5$ & $-5.45 \pm 0.3$ & $78 \pm 2.3$ & $88.2 \pm 0.9$ & 81.0 \\
\hline F8 & $34 \pm 1.7$ & $477 \pm 1.07$ & $-5.18 \pm 0.2$ & $87 \pm 2.0$ & $95.3 \pm 0.8$ & 90.1 \\
\hline F9 & $17 \pm 1.5$ & $47 I \pm 3.6$ & $-6.84 \pm 0.1$ & $83 \pm 1.9$ & $96.0 \pm 1.7$ & 86.9 \\
\hline FI9* & $27 \pm 0.8$ & $336 \pm 11.6$ & $-15.4 \pm 0.6$ & $94 \pm 1.1$ & $100 \pm 0.6$ & 95.5 \\
\hline F20 & $23 \pm 0.6$ & $380 \pm 5.6$ & $-11.9 \pm 0.2$ & $79 \pm 2.2$ & $95.5 \pm 2.1$ & 88.1 \\
\hline F2I & $32 \pm 1.3$ & $423 \pm 4.4$ & $-8.6 \pm 0.03$ & $78 \pm 0.9$ & $94.0 \pm 1.7$ & 85.4 \\
\hline F26 & $75 \pm 3.2$ & $823 \pm 3.2$ & $-3.7 \pm 0.5$ & $77 \pm 3.1$ & $85.0 \pm 2.8$ & 82.5 \\
\hline F27 & $99 \pm 2.7$ & $775 \pm 6.2$ & $-2.2 \pm 0.4$ & $73 \pm 2.5$ & $78.7 \pm 2.3$ & 73.5 \\
\hline Pure drug & NA & NA & NA & $19 \pm 1.3$ & $55 \pm 2.2$ & 42.5 \\
\hline
\end{tabular}

Notes: Data are presented as mean \pm standard deviation. *FI9 is the optimal formula that was selected for in vivo studies. Abbreviation: NA, not applied.

$10 \% \mathrm{w} / \mathrm{w}$ oil was $420 \mathrm{~nm}$ while that of $\mathrm{F} 27$ with $30 \%$ oil was $775 \mathrm{~nm}$. At the same oil concentration, increasing the amount of surfactant concentration produced droplets with smaller mean size as in case of $\mathrm{F} 4$ with surfactant:cosurfactant ratio of 4:1 that showed the least droplet size of $285 \mathrm{~nm}$.

The zeta potential results of the diluted SEDDS formulations are expressed as mean $\pm \mathrm{SD}$ and shown in Table 3. All formulations showed moderate negative zeta potential. Diluted SEDDS formulations F4 and F19 have a significantly higher absolute zeta potential than the other formulations.

\section{In vitro release studies}

In vitro drug release studies were performed for SEDDS formulations that passed the stability tests and pure unprocessed drug as control. The results are presented as the percentage cumulative drug released vs time plots (Figure 2). The dissolution parameters are expressed as amount of drug dissolved in the first 5 minutes (Q5) and at the end of the experiment (Q60), and percentage dissolution efficiency (\%DE) (Table 3). The \%DE of a pharmaceutical dosage form is defined as the area under the dissolution curve up to a certain time, $t$, expressed as a percentage of the area of the rectangle described by $100 \%$ dissolution at the same time. ${ }^{26}$ The $\% \mathrm{DE}$ can be calculated from the following equation:

$$
D E=\frac{\int_{0}^{t} y \times d t}{y 100 \times t} \times 100
$$

The release pattern of the unprocessed drug was slow with Q5 of 19\% of the labeled amount, followed by slow release with Q60 of only $55 \%$ and low dissolution efficiency of $42 \%$. Incorporation of the drug in SEDDS significantly improved dissolution rate as reflected by increased dissolution parameters from all formulations. The amount released in the first 5 minutes ranged from $73 \%$ to $93 \%$ with overall dissolution efficiency of 73.5-100 in case of F27 and F19, respectively. There was a similar drug release pattern for formulations prepared using $10 \%, 15 \%$, and $20 \%$ oil $(P>0.05)$; however, they showed a significant enhancement compared to those with $30 \%$ oil. Formulations F4 and F19 showed the most pronounced drug release properties compared to the other SEDDS formulations, while F27 showed the least drug release.

\section{In vivo study}

\section{Determination of blood glucose level and body weight}

The body weight and blood glucose levels determined at the onset and end of the experiment are presented in Table 4. It can be observed that the blood glucose levels and body weight at the onset of the study showed no significant differences $(P>0.05)$ between the tested groups. By the end of the experiment, blood glucose level was significantly increased, while the body weight was significantly decreased in diabetic (DM) group $(P<0.05)$ when compared to the normal control group. Nevertheless, treatment with resveratrol had highly significantly $(P<0.001)$ ameliorated effects on glucose 
A

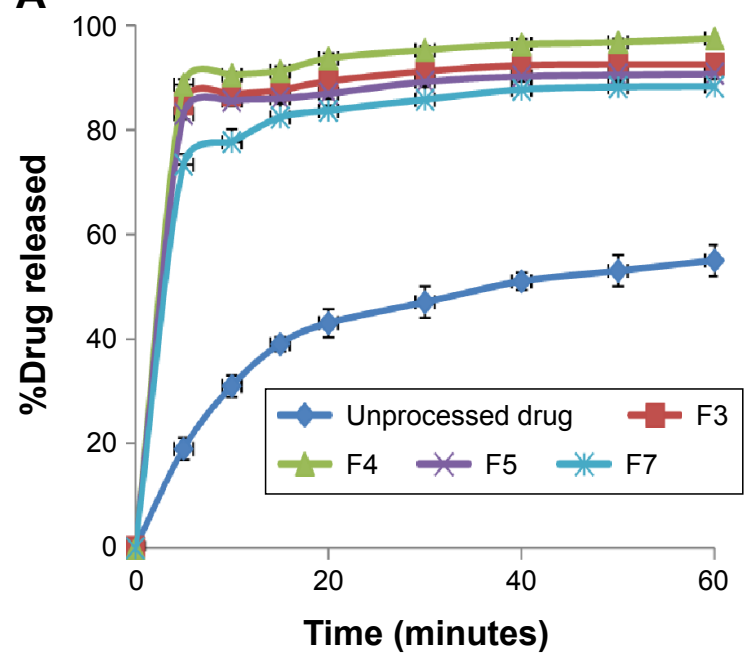

C

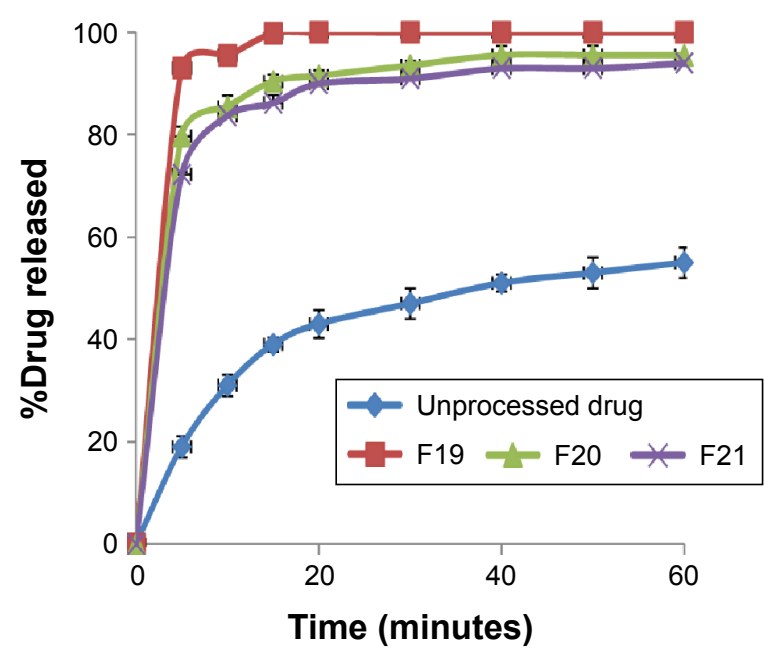

B

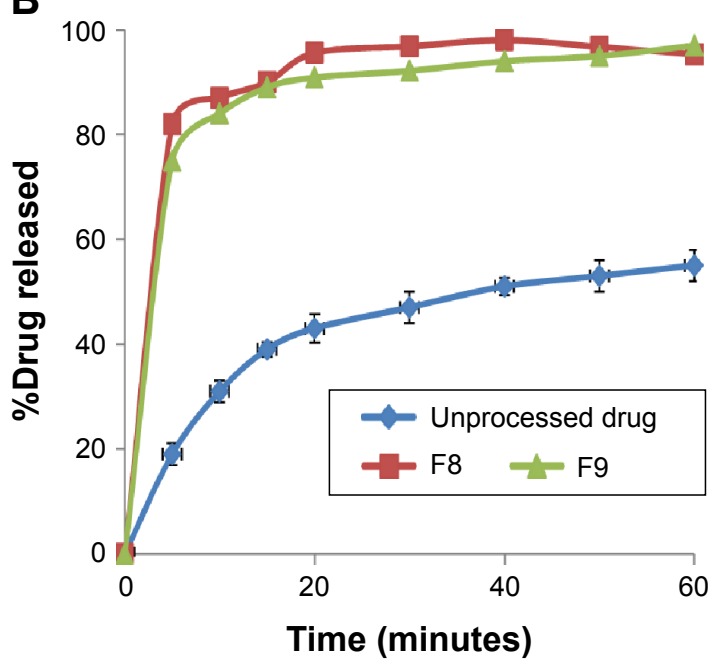

D

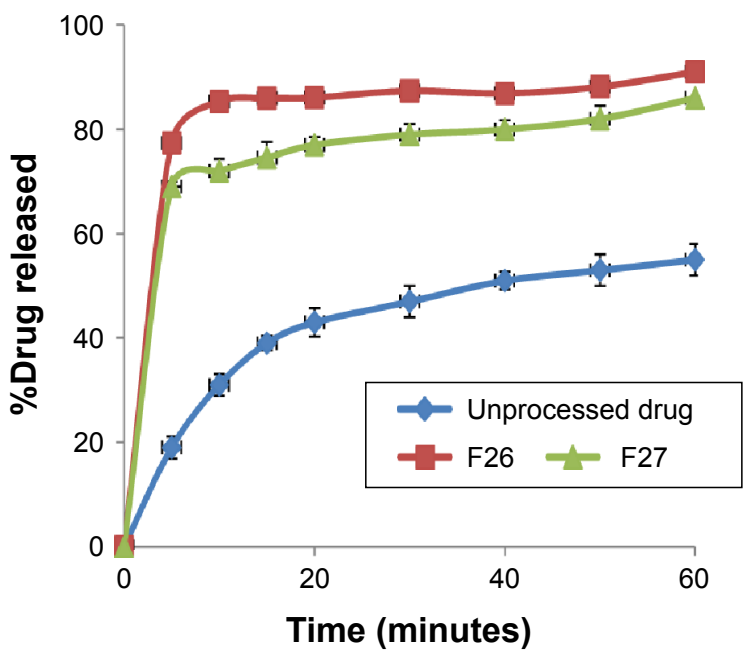

Figure 2 Dissolution profiles of resveratrol from different self-emulsifying drug delivery system formulations prepared using different oil ratios of I0\% (A), I5\% (B), $20 \%$ (C), and $30 \%($ D).

Table 4 The effect of orally administered optimized resveratrol SEDDS (FI9) compared with unprocessed resveratrol on body weight and serum glucose level in streptozotocin-induced diabetes mellitus in male albino rats

\begin{tabular}{|c|c|c|c|c|}
\hline \multirow[t]{2}{*}{ Group } & \multicolumn{2}{|c|}{ Body weight (g) } & \multicolumn{2}{|c|}{ Serum glucose level (mg/dL) } \\
\hline & $\begin{array}{l}\text { Before } \\
\text { treatment }\end{array}$ & $\begin{array}{l}\text { After } 4 \text { weeks } \\
\text { treatment }\end{array}$ & At the onset & $\begin{array}{l}\text { At the end of } \\
\text { experiment }\end{array}$ \\
\hline$N$ & $195.12 \pm 3.9$ & $219.9 \pm 8.81$ & $95.5 \pm 3.6$ & $106.3 \pm 3.4$ \\
\hline DM & $189.6 \pm 7.1$ & $\mid 29.6 \pm 4.01 *$ & $296.8 \pm 9.1 *$ & $449.8 \pm 7.9 *$ \\
\hline CRVIO & $194.0 \pm 5.6$ & $168.25 \pm 2.8^{* * * *}$ & $282.8 \pm 7.6^{*}$ & $196.4 \pm 6.74 * * *$ \\
\hline FRVIO & $180.8 \pm 3.92$ & $185.6 \pm 4.98 *, * *, * * *$ & $266.35 \pm 5^{* * * *}$ & $179.7 \pm 3.92^{* * * * * * *}$ \\
\hline CRV20 & $192.8 \pm 8.9$ & $|88.5 \pm| 3.4^{* *, * * *}$ & $272.3 \pm 4.4^{* * * *}$ & $170.5 \pm 3.43^{*, * * * * * *}$ \\
\hline FRV20 & $196.6 \pm 7.01$ & $190.2 \pm 4.8 * *, * * *$ & $267.6 . \pm 4.8^{*}$ & $172.3 \pm 4.15^{*, * *, * * *}$ \\
\hline
\end{tabular}

Notes: Values were expressed as mean \pm standard error $(n=10$ per group). P-value is $<0.05$; *significant difference vs $\mathrm{N}$ group; **significant difference vs DM group; and ****significant vs CRVIO.

Abbreviations: $\mathrm{N}$, nondiabetic control; DM, streptozotocin-induced diabetes; CRVIO, DM treated with unprocessed resveratrol ( $10 \mathrm{mg} / \mathrm{kg} / \mathrm{day})$; FRVIO, DM treated with optimal formula of resveratrol SEDDS (FI9) (10 mg/kg/day); CRV20, DM treated with unprocessed resveratrol $(20 \mathrm{mg} / \mathrm{kg} / \mathrm{day})$; FRV20, DM treated with optimal formula of resveratrol SEDDS (FI9) $(20 \mathrm{mg} / \mathrm{kg} /$ day); SEDDS, self-emulsifying drug delivery system. 


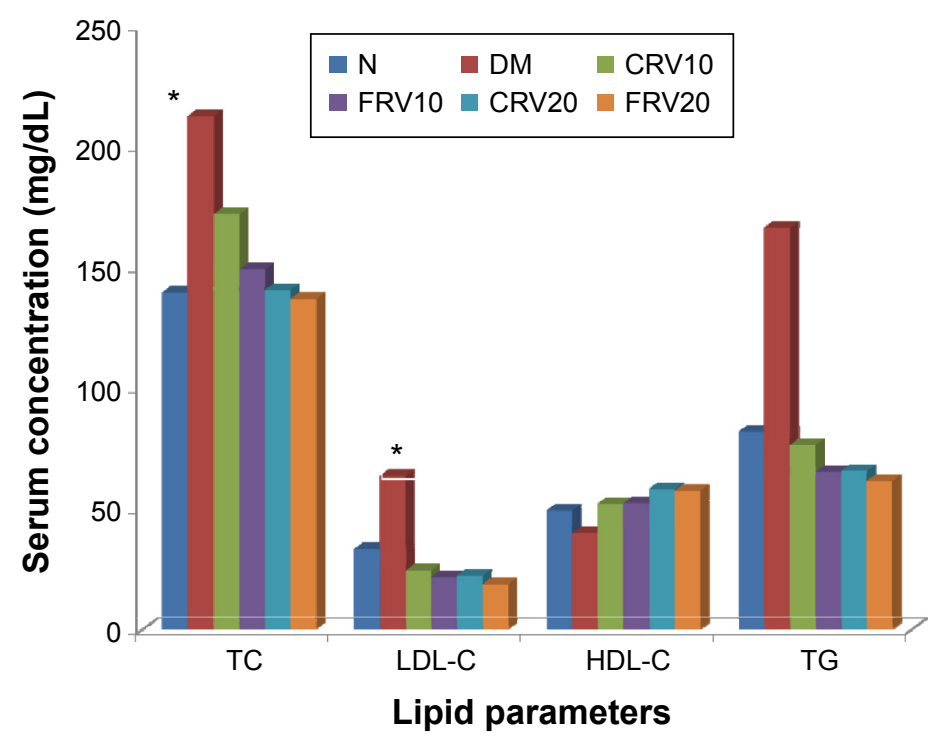

Figure 3 Effect of orally administered optimized resveratrol SEDDS (FI9) compared with unprocessed resveratrol on serum lipid levels in streptozotocin-induced diabetes mellitus in male albino rats.

Notes: Values were expressed as mean \pm standard error ( $n=10$ per group). $P$-value is $<0.05$; *significant difference vs $N$ group.

Abbreviations: $\mathrm{N}$, nondiabetic control; DM, streptozotocin-induced diabetes; CRVI0, DM treated with unprocessed resveratrol (I0 mg/kg/day); FRVI0, DM treated with optimal formula of resveratrol SEDDS (FI9) (10 mg/kg/day); CRV20, DM treated with unprocessed resveratrol (20 mg/kg/day); FRV20, DM treated with optimal formula of resveratrol SEDDS (FI9) (20 mg/kg/day); SEDDS, self-emulsifying drug delivery system; TC, total cholesterol; LDL-C, low density lipoprotein-cholesterol; HDL-C, high density lipoprotein-cholesterol; TG, triglycerides.

level and body weight in the FRV10, CRV20, and FRV20 groups when compared to the DM group. However, moderate significant $(P<0.05)$ differences in glucose level and body weight were observed when unprocessed resveratrol (10 $\mathrm{mg} / \mathrm{kg}$ ) was given in CRV10 group in comparison to the nontreated diabetic DM group.

\section{Biochemical analysis}

The results revealed that serum total cholesterol, LDL-C, and triglycerides levels were significantly increased while HDL-C significantly decreased in the untreated diabetic DM group when compared to the normal control group $(P<0.05)$ (Figure 3). However, treatment with resveratrol (unprocessed/or SEDDS formulation) prevented the increase of total cholesterol, LDL-cholesterol, and triglyceride levels and ameliorated the reduction of HDL-C in all diabetic treated groups $(P<0.05)$.

\section{Discussion}

\section{Saturation solubility}

One important consideration when formulating a selfemulsifying system is avoiding precipitation of the drug. Therefore, the components used in the system should have high solubilizing capacity for the drug to ensure solubilization of the drug in the resultant dispersion. In the present study, olive oil was chosen as oil, Tween 80 as surfactant, and propylene glycol as cosurfactant. Increased drug solubility in crude oils compared to oleic acid can be attributed to their composition which has a mixture of unsaturated fatty acids. This mixture can provide a cosolvent effect. The relative efficacy of olive oil over soybean and almond can be due to the relative composition of fatty acids which can affect the cosolvency properties. Tween 80 , a hydrophilic nonionic surfactant of hydrophilic/lipophilic balance $($ HLB) $=15$, was found to have the maximum solubilizing capacity which is required for providing fine, uniform emulsion droplets. ${ }^{27}$ Presence of cosurfactant is important in the formulation of SEDDS as it decreases the bending strain at the $\mathrm{o} / \mathrm{w}$ interface and increases the flexibility of the interfacial film in addition to increasing the solubility of the lipophilic drug in the system.

\section{Phase diagram and self-emulsification assessment}

One of the most important characteristics of SEDDS is the change that occurs when the system is diluted as it will be diluted by body fluids after administration. Therefore, ternary phase diagram was constructed to identify self-emulsifying regions and select the most suitable concentrations of oil, surfactant, and cosurfactant for the formulation of SEDDS with good stability. ${ }^{28} \mathrm{~A}$ fixed volume ratio of water to liquid SEDDS (500:1) was used to evaluate the self-emulsifying behavior in view of the fact that the basic volume of gastric 
juice $(10-100 \mathrm{~mL})$ is hundred times larger than the total amount of SEDDS. Since SEDDS formed fine o/w emulsions with only gentle agitation upon introduction into aqueous media, the free energy required to form an emulsion was very low and the formulation was thermodynamically spontaneous. ${ }^{29}$ All emulsions were stable at zero time and this may be due to the high hydrophilicity of Tween 80 (HLB =15) and high solubilizing capacity of propylene glycol. It was stated that self-emulsification occurs when the entropy change that favors dispersion is greater than the energy required to increase the surface area of the dispersion. ${ }^{30}$ It was also reported that cosurfactant fluidizes hydrocarbon region of the interfacial film decreasing the bending strain of the film and facilitating the reduction of interfacial tension. ${ }^{27}$ It was observed that self-emulsification process is highly affected by the $S_{\text {mix }}$ ratio and oil content. Similar results were reported by Derle et al..$^{31}$ In addition, increasing oil proportion resulted in a decrease in the clarity of emulsion. This could be attributed to the increased interfacial tension between oil and aqueous phase as a result of insufficient concentration of surfactant/cosurfactant system. ${ }^{27}$

\section{Characterization}

\section{Thermodynamic stability studies}

It is the thermodynamic stability which differentiates nanoor microemulsion from emulsions that have kinetic stability and will eventually phase separate. The improved thermodynamic stability of most SEDDSs could be attributed to the preferential adsorption of both surfactant and cosurfactant at the $\mathrm{o} / \mathrm{w}$ interface, thereby reducing the interfacial energy required for zeroing coalescence. ${ }^{32}$ The instability of the formulations F17, F25, and F28 may be due to variation in the concentration of different components.

\section{Emulsification time and precipitation assessment}

The rate of emulsification is an important index for the assessment of the efficiency of emulsification. The SEDDS should disperse completely and quickly when subjected to aqueous media under mild agitation as the free energy required to form an emulsion is very low and, therefore, the emulsification is thermodynamically spontaneous. ${ }^{33}$ The visual observations indicated that the higher $\mathrm{S}_{\text {mix }}$, the greater the spontaneity of emulsification. This may be due to the efficiency of Tween 80 to lower the interfacial tension at $\mathrm{o} / \mathrm{w}$ interface, as the presence of high concentration of surfactant will facilitate the self-emulsification process and eventually lead to rapid emulsification rate. Additionally, cosurfactant would augment the reduction to the interfacial tension and also influence interfacial film curvature and hence spontaneity of emulsification process..$^{34,35}$ The investigated SEDDS formulations resulted in the formation of stable microemulsions upon dilution and hence they were selected for further investigation.

\section{Determination of droplet size and zeta potential}

The results revealed that surfactant concentration plays a vital role in the nanoemulsification process. This could be due to the availability of optimum concentration of surfactant system to stabilize $\mathrm{o} / \mathrm{w}$ interface and form a better closed pack film at o/w interface leading to rapid maturation of the droplets which subsequently results in smaller droplet size. ${ }^{34}$

It is well accepted that emulsion stability is directly related to the magnitude of the surface charge that would prevent droplet coalescence upon random collisions of the colloidal dispersion leading to repulsion forces that would stabilize the formulation. ${ }^{36}$ The significantly highest absolute zeta potential of the formulations F4 and F19 could be a result of their small droplet size which has more surface area and more charge density. Thus, upon emulsification, these formulations would generate a more stable emulsion than the other SEDDSs. The formulation F19 was selected instead of F4, though the later showed smaller droplet size due to the higher value of zeta potential that would improve stability by preventing globular coalescence. Additionally, F19 contains higher oil concentration that would otherwise improve uptake by the lymphatic drainage.

\section{In vitro release studies}

Poor drug dissolution that largely contributes to the poor oral bioavailability of resveratrol may be ascribed to the presence of the drug in the crystalline form that dissolves slowly in the aqueous medium. The enhanced drug dissolution pattern from different SEDDS formulations could be attributed to the fact that SEDDS is dispersed in the dissolution medium forming microemulsion with small droplet size and thus large surface area into which the drug can be dissolved. Similar findings were obtained by Bhikshapathi et al who reported that in SEDDS the drug will be in dissolved form whereas pure drug will be in a crystalline form and, hence, more time will be needed for drug release from the latter. ${ }^{33}$ The improved release properties from the formulations F4 and F19 may be ascribed to proper balance between the percentage of oil-surfactant mixture results in a decreased droplet size that reflects increased surface area. ${ }^{37}$ On the other hand, least dissolution results from F27 may be due to insufficient proportion of surfactant system $\left(\mathrm{S}_{\text {mix }}=1: 3\right)$ 
with a high proportion of oil $(30 \% \mathrm{w} / \mathrm{w})$, which led to lesser reduction in interfacial tension between oil and aqueous phase followed by decreased drug partition from large droplets into aqueous medium. ${ }^{38}$

As stated before, both F4 and F19 showed a similar results regarding droplet size and release behavior. Interestingly, though F4 showed the least particle size, F19 gave a higher Q5 and dissolution efficiency of $96 \%$ compared to $89 \%$ of the former. This could be explained by the fact that F4 contains a larger amount of surfactant, which though reducing the particle size would affect drug dissolution rate. The possible mechanism is the high concentration of surfactant would otherwise impede the diffusion path of the drug due to higher viscosity. In addition, F19 contains high amount of propylene glycol that, as cosurfactant, would increase interfacial fluidity by penetrating into the surfactant film creating void space between surfactant molecules thus increasing drug diffusion out of globules. ${ }^{17}$

As oil is considered the main excipient of SEDDS because it can increase the fraction of lipophilic drug transported via the intestinal lymphatic system with subsequent increase in absorption from the GIT, ${ }^{21}$ F19 (20\% oil) was selected as the best formula and underwent in vivo studies.

\section{In vivo study}

\section{Determination of blood glucose level and body} weight

The results revealed that the elevated blood glucose level and reduced body weight in diabetic rats were significantly ameliorated in all resveratrol treated groups with high efficacy of FRV10 than unprocessed CRV10 and comparable efficacy to higher doses (CRV20 and FRV20). The results of blood glucose level and body weight were in accordance with several studies that reported anti-hyperglycemic effect of resveratrol. ${ }^{39,40}$ It was supposed that resveratrol ameliorates diabetes-mediated hyperglycemia through insulin-dependent and insulin-independent pathways. ${ }^{29}$ Szkudelski and Szkudelska reported that resveratrol preserves the pancreatic $\beta$-cells against chronic overstimulation leading to insulin secretion ability. ${ }^{7}$ In addition, Miura et a ${ }^{41}$ suggested a possible recovery mechanism of resveratrol on the remnant $\beta$-cell functionality and partially improved dyslipidemia in experimental diabetic animals. On the other hand, it has been shown that resveratrol increases the translocation of Glut-4 and glucose uptake in diabetic peripheral tissues through activation of some intracellular signaling components via an insulin-independent manner. ${ }^{42}$ Improvement in glucose metabolism in turn prevents degradation of proteins and lipids from their reservoirs and attenuates the body weight loss during diabetes. ${ }^{30}$ Improvement in insulin sensitivity after 4-week resveratrol administration also has been recently reported in type 2 diabetic patients as well as in adults with impaired glucose tolerance. ${ }^{43,44}$

Insufficient secretion or action of insulin causes hyperglycemia, mainly via an enhanced release of glucose by the liver and reduced utilization of glucose in peripheral tissues. In this situation, the body has to provide itself energy by degradation of proteins and lipids from their reservoirs, which ultimately account for diabetes-induced weight loss. ${ }^{45}$ The results revealed that treatment with resveratrol attenuated weight loss process suggesting possible improvement in energy metabolism. This observation is in agreement with previously published literatures..$^{27,45}$ Moreover, observed efficacy of the lower dose of resveratrol SEDDS (group FRV10) is comparable to the higher doses (groups CRV20 and FRV20), which may be attributed to increased resveratrol bioavailability by SEDDS formulation. Lipid is an essential component of the SEDDS formulation and hence it can solubilize marked amounts of lipophilic drug, facilitate self-emulsification, and has the propensity to augment the fraction of drug transported via intestinal lymphatic system thereby increasing its absorption from the gastrointestinal tract and bioavailability becomes amplified. ${ }^{2}$

\section{Biochemical analysis}

Diabetes is usually associated with profound complications of plasma lipid and lipoprotein profile. There are many reports that suggested the controversial effect of resveratrol on lipid profile in experimental animals. The results revealed that treatment with resveratrol (unprocessed or SEDDS formulation) prevented the increase of total cholesterol, LDL-cholesterol, and triglyceride levels and ameliorated the reduction of HDL-C in diabetic rats. Although improvement of lipid profile was better in groups FRV10, FRV20, and CRV20 than in group CRV10, statistical analysis of the results demonstrated no significant difference between all treated groups.

A previous study reported that resveratrol with a dosage of $10 \mathrm{mg} / \mathrm{kg}$ administered intragastrically for 8 weeks lowered triglyceride level. ${ }^{46}$ It has been anticipated that resveratrol activates 5'-AMP-activated protein kinase which suppresses fatty acid synthesis and inhibits acetyl-CoA carboxylase elevating their oxidation.

In another study, Do et $\mathrm{al}^{47}$ examined the long-term effect of resveratrol supplementation on suppression of atherogenic lesion formation and cholesterol synthesis and 
LDL-C oxidation in apo E-deficient mice. It was found that the concentration of LDL-C and total cholesterol was significantly decreased in the resveratrol treated groups compared to the control group. Kim et al suggested that resveratrol may hinder adipogenesis possibly through suppression of the galanin-mediated signaling pathway, pro-inflammatory signaling cascades, and cytokine production in adipose tissue of mice fed a high fat diet. ${ }^{48}$

\section{Conclusion}

The present study demonstrated the potential usefulness of SEDDS in the improvement of dissolution rate and thereby oral bioavailability of resveratrol compared to unprocessed drug. SEDDS formed from olive oil, Tween 80, and propylene glycol in the ratio 200:266.7:533.3 (in $\mathrm{mg}$ ) appeared to be an interesting approach to improve problems associated with oral delivery of resveratrol optimization. The in vivo investigation using this formula reflected a potential hypoglycemic and hypolipidemic effect of resveratrol. Moreover, there was no significant difference between low $(10 \mathrm{mg})$ and high $(20 \mathrm{mg})$ doses of resveratrol SEDDS in reducing the blood glucose and body weight in STZ- induced diabetic rats suggesting high efficiency of resveratrol SEDDS.

\section{Acknowledgment}

The authors are grateful for financial support from the Pharmaceutical Sciences Research Centre, Umm Al-Qura University, Makkah, KSA (Project No 4331013).

\section{Disclosure}

The authors report no conflicts of interest in this work.

\section{References}

1. Farnsworth NR, Akerele O, Bingel AS, Soejarto DD, Guo Z. Medicinal plants in therapy. Bull WHO. 1985;63:965-981.

2. Amri A, Chaumeil JC, Sfar S, Charrueau C. Administration of resveratrol: What formulation solutions to bioavailability limitations? J Control Release. 2012;158:182-193.

3. Ansari KA, Vavia PR, Trotta F, Cavalli R. Cyclodextrin-based nanosponges for delivery of Resveratrol: In vitro characterisation, stability, cytotoxicity and permeation study. AAPS Pharm Sci Tech. 2011;12(1):279-286.

4. Das S, Das DK. Resveratrol: a therapeutic promise for cardiovascular diseases. Recent Pat Cardiovasc Drug Discov. 2007;2:133-138.

5. Hung LM, Su MJ, Chen JK. Resveratrol protects myocardial ischemiareperfusion injury through both NO-dependent and NO-independent mechanisms. Free Radic Biol Med. 2004;36:774-781.

6. Baur JA, Sinclair DA. Therapeutic potential of resveratrol: the in vivo evidence. Nat Rev Drug Discov. 2006;5(6):493-506.

7. Szkudelski T, Szkudelska K. Anti-diabetic effects of resveratrol. Ann NY Acad Sci. 2011;1215:34-39.

8. Hausenblas HA, Schoulda JA, Smoliga JM. Resveratrol treatment as an adjunct to pharmacological management in type 2 diabetes mellitus systematic review and meta-analysis. Mol Nutr Food Res. 2015;59(1): $147-159$.
9. Boocock DJ, Faust GES, Patel KR, et al. Phase I dose escalation pharmacokinetic study in healthy volunteers of resveratrol, a potential cancer chemopreventive agent. Cancer Epidemiol Biomarkers Prev. 2007;16(6):1246-1252.

10. Kristl J, Teskac K, Caddeo C, Abramović Z, Sentjurc M. Improvement of cellular stress response on Resveratrol in liposomes. Eur J Pharm Biopharm. 2009;73(2):253-259.

11. Amidon GL, Lennernäs H, Shah VP, Crison JR. A theoretical basis for a biopharmaceutic drug classification: the correlation of in vitro drug product dissolution and in vivo bioavailability. Pharm Res. 1995;12(3): 413-420.

12. López-Nicolás JM, Núñez-Delicado E, Pérez-López AJ, Barrachina AC, Cuadra-Crespo P. Determination of stoichiometric coefficients and apparent formation constants for $\beta$-cyclodextrin complexes of trans-resveratrol using reversed-phase liquid chromatography. J Chromatogr A. 2006;1135(2):158-165.

13. Gokce EH, Korkmaz E, Dellera E, Sandri G, Bonferoni MC, Ozer O. Resveratrol-loaded solid lipid nanoparticles versus nanostructured lipid carriers: evaluation of antioxidant potential for dermal applications. Int J Nanomed. 2012;7:1841-1850.

14. Atanacković MT, Gojković-Bukarica LC, Cvejić JM. Improving the low solubility of resveratrol. Presented at: 18th Scientific Symposium of the Austrian Pharmacological Society (APHAR) joint meeting with the Croatian, Serbian and Slovenian Pharmacological Societies; September 20-21, 2012; Graz, Austria.

15. Bhattacharyya A, Bajpai M. Development and evaluation of a selfemulsifying drug delivery system of amphotericin B. Asian J Pharm. 2012;6(2):124-129.

16. Sapra K, Sapra A, Singh SK, Kakkar S. Self emulsifying drug delivery system: A tool in solubility enhancement of poorly soluble drugs. Indo Global J Pharm Sci. 2012;2(3):313-332.

17. Charman SA, Charman WN, Rogge MC, Wilson TD, Dutko FJ, Pouton CW. Self emulsifying drug delivery systems: formulation and biopharmaceutical evaluation of an investigational lipophilic compound. Pharm Res. 1992;9(1):87-93.

18. Balakrishnan P, Lee B, Oh DH, et al. Enhanced oral bioavailability of dexibuprofen by a novel solid Self-emulsifying drug delivery system (SEDDS). Eur J Pharm Biopharm. 2009;72(3):539-545.

19. Mei X, Etzler FM, Wang Z. Use of texture analysis to study hydrophilic solvent effects on the mechanical properties of hard gelatin capsules. Int J Pharm. 2006;324(2):128-135.

20. Harshal M, Tanvir S, Dheeraj B, Rajendra W. Design and development of solid self-micro-emulsifying drug delivery system (SEDDS) of fenofibrate. Int J Pharm Pharm Sci. 2011;3(4):163-166.

21. Shah NH, Carvajal MT, Patel CI, Infeld MH, Malick AW. Self-emulsifying drug delivery systems (SEDDS) with polyglycolysed glycerides for improving in vitro dissolution and oral absorption of lipophilic drugs. Int J Pharm. 1994;106(1):15-23.

22. Society of Toxicology. Guiding Principles in the Use of Animals in Toxicology. Reston, VA: Society of Toxicology; 2008. Available from: http://www.toxicology.org/AI/FA/guidingprinciples.pdf. Accessed August 6, 2013.

23. Schmatz R, Mazzanti CM, Spanevello R, et al. Ectonucleotidase and acetylcholinesterase activities in synaptosomes from the cerebral cortex of streptozotocin-induced diabetic rats and treated with resveratrol. Brain Res Bull. 2009;80(6):371-376.

24. Lee ES, Shin MO, Yoon S, Moon JO. Resveratrol inhibits dimethylnitrosamine-induced hepatic fibrosis in rats. Arch Pharm Res. 2010;33(6): 925-932.

25. Friedewald WT, Levy RI, Fredrickson DS. Estimation of the concentration of low-density lipoprotein cholesterol in plasma, without use of the preparative ultracentrifuge. Clin Chem. 1972;18(6):499-502.

26. Biswal S, Sahoo J, Murthy PN, Giradkar RP, Avari JG. Enhancement of dissolution rate of gliclazide using solid dispersions with polyethylene glycol 6000. AAPS Pharm Sci Tech. 2008;9(2):563-570.

27. Kallakunta VR, Bandari S, Jukanti R, Veerareddy PR. Oral self emulsifying powder of lercanidipine hydrochloride: Formulation and evaluation. Powd Tech. 2012;221:375-382. 
28. Patel AR, Vavia PR. Preparation and in vivo evaluation of SMEDDS (self microemulsifying drug delivery system) containing fenofibrate. AAPS J. 2007;9(3):E344-E352.

29. Kassem AA, Marzouk MA, Ammar AA, Elosaily GH. Preparation and in vitro evaluation of self-nanoemulsifying drug delivery systems (SNEDDS) containing clotrimazole. Drug Discov Ther. 2010;4(5): 373-379.

30. Reiss H. Entropy-induced dispersion of bulk liquids. J Colloids Interface Sci. 1975;53(1):61-70.

31. Derle DV, Sagar BSH, Pimpale S. Microemulsion as a vehicle for transdermal permeation of nimesulide. Ind J Pharm Sci. 2006;68(5): $622-625$.

32. Rao SVR, Shao J. Self-nanoemulsifying drug delivery systems (SNEDDS) for oral delivery of protein drugs. I. Formulation development. Int J Pharm. 2008;362(1-2):2-9.

33. Bhikshapathi D, Madhukar P, Kumar BD, Kumar GA. Formulation and characterization of pioglitazone $\mathrm{HCl}$ self emulsifying drug delivery system. Der Pharmacia Let. 2013;5(2):292-305.

34. Gursoy RN, Benita S. Self-emulsifying drug delivery systems (SEDDS) for improved oral delivery of lipophilic drugs. Biomed Pharmacother. 2004;58(3):173-182.

35. Khan F, Islam S, Roni MA, Jalil R. Systematic development of selfemulsifying drug delivery systems of atorvastatin with improved bioavailability. Potential Sci Pharm. 2012;80(4):1027-1043.

36. Suresh PK, Sharma S. Formulation and in-vitro characterization of self-nanoemulsifying drug delivery system of cinnarizine. Int J Compr Pharm. 2011;9(8):1-6.

37. Burtis CA, Ashwood ER. Carbohydrates. In: Saunders WB, editor. Tietz Textbook of Clinical Chemistry. Philadelphia, Pa, USA; 1999: 750-808.

38. Kallakunta VR, Eedara BB, Jukanti R, Ajmeera RK, Suresh Bandari S. A Gelucire 44/14 and labrasol based solid self emulsifying drug delivery system: formulation and evaluation. J Pharm Invest. 2013;43: 185-196.

39. Turan B, Tuncay E, Vassort G. Resveratrol and diabetic cardiac function: focus on recent in vitro and in vivo studies. J Bioenerg Biomembr. 2012;44(2):281-296.
40. Soufi FG, Vardyani M, Sheervalilou R, Mohammadi M, Somi MH. Long-term treatment with resveratrol attenuates oxidative stress proinflammatory mediators and apoptosis in streptozotocin-nicotinamideinduced diabetic rats. Gen Physiol Biophys. 2012;31(4):431-438.

41. Miura D, Miura Y, Yagasaki K. Hypolipidemic action of dietary resveratrol, a phytoalexin in grapes and red wine, in hepatoma-bearing rats. Life Sci. 2003;73(11):1393-1400.

42. Penumathsa SV, Thirunavukkarasu M, Zhan L, et al. Resveratrol enhances GLUT-4 translocation to the caveolar lipid raft fractions through AMPK/Akt/eNOS signalling pathway in diabetic myocardium. J Cell Mol Med. 2008;12:2350-2361.

43. Brasnyo P, Molnar GA, Mohas M, et al. Resveratrol improves insulin sensitivity, reduces oxidative stress and activates the Akt pathway in type 2 diabetic patients. Br J Nutr. 2011;106(3):383-389.

44. Crandall JP, Oram V, Trandafirescu G, et al. Pilot study of resveratrol in older adults with impaired glucose tolerance. J Gerontol A Biol Sci Med Sci. 2012;67(12):1307-1312.

45. Palsamy P, Subramanian S. Ameliorative potential of resveratrol on proinflammatory cytokines, hyperglycemia mediated oxidative stress, and pancreatic $b$-cell dysfunction in streptozotocin-nicotinamideinduced diabetic rats. J Cell Physiol. 2010;224(2):423-432.

46. Rivera L, Moron R, Zarzuelo A, Galisteo M. Long-term resveratrol administration reduces metabolic disturbances and lowers blood pressure in obese Zucker rats. Biochem Pharmacol. 2009;77(6):1053-1063.

47. Do GM, Kwon EY, Kim HJ, Jeon SM, Ha TY, Park T. Long-term effects of resveratrol supplementation on suppression of atherogenic lesion formation and cholesterol synthesis in apo E-deficient mice. Biochem Biophys Res Commun. 2008;374(1):55-59.

48. Kim S, Jin Y, Choi Y, Park T. Resveratrol exerts anti-obesity effects via mechanisms involving down-regulation of adipogenic and inflammatory processes in mice. Biochem Pharmacol. 2011;81: 1343-1351.
Drug Design, Development and Therapy

\section{Publish your work in this journal}

Drug Design, Development and Therapy is an international, peerreviewed open-access journal that spans the spectrum of drug design and development through to clinical applications. Clinical outcomes, patient safety, and programs for the development and effective, safe, and sustained use of medicines are a feature of the journal, which

\section{Dovepress}

has also been accepted for indexing on PubMed Central. The manuscript management system is completely online and includes a very quick and fair peer-review system, which is all easy to use. Visit http://www.dovepress.com/testimonials.php to read real quotes from published authors. 